HOT TOPICS

\title{
A prefrontal social circuit vulnerable to juvenile social isolation
}

\author{
Hirofumi Morishita (iD) 1,2,3,4,5,6
}

Neuropsychopharmacology (2021) 46:229-230; https://doi.org/10.1038/s41386-020-00821-6

Loneliness is becoming increasingly recognized as a threat to mental health [1]. Social isolation during childhood is particularly detrimental to the normal functioning of the medial prefrontal cortex (mPFC) and the establishment of adult social behavior. In mice, juvenile social isolation (jSI) following weaning for 2 weeks, but not during a later period, leads to decreased sociability in adult male mice, suggesting a juvenile sensitive period for establishing adult sociability [2, 3]. Identifying a specific mPFC social circuit vulnerable to childhood isolation will likely point toward therapeutic targets for the amelioration of social processing deficits shared across a range of psychiatric disorders [4].

In our recent studies [3, 5], we identified specific subpopulations of mPFC excitatory and inhibitory neurons that are required for normal sociability and profoundly vulnerable to $\mathrm{jSI}$ in mice (Fig. 1). Among excitatory pyramidal neurons, we found that mPFC neurons projecting to the posterior paraventricular thalamus (PVT), which relays signals to the classical reward circuitry, are activated during social exploration in adult grouphoused mice, but not in jSI mice [5]. Chemogenetic or optogenetic suppression of mPFC $\rightarrow$ PVT activity is sufficient to induce sociability deficits in group-housed mice. jSI leads to both reduced excitability of $\mathrm{mPFC} \rightarrow$ PVT neurons and increased inhibitory input drive in adulthood. In contrast, other MPFC neurons projecting to nucleus accumbens or contralateral mPFC show no deficits in jSI mice $[5,6]$, suggesting that $\mathrm{mPFC} \rightarrow \mathrm{PVT}$ neurons and the associated inhibitory neurons are particularly vulnerable to jSI.

On inhibitory neurons' side, we found that a specific subclass of deep-layer Somatostatin-expressing low-threshold spiking interneurons (LTS-SSTIs) showed increased excitability [5], likely contributing to an increased inhibitory drive onto $\mathrm{mPFC} \rightarrow \mathrm{PVT}$ neurons in jSI mice. In contrast, Parvalbumin-expressing interneurons (PVIs) show reduced intrinsic excitability and decreased synaptic drive [3] in a similar fashion to $\mathrm{mPFC} \rightarrow$ PVT neurons in adult jSI mice. These divergent changes in mPFC interneurons seem to contribute to sociability deficits in jSI mice as chemogenetic suppression of mPFC PVIs or activation of LTSSSTIs recapitulates sociability deficits caused by jSI [3, 5]. To our surprise, these deficits are not observed immediately after the isolation period but only later, in adulthood. This suggests that the
A.

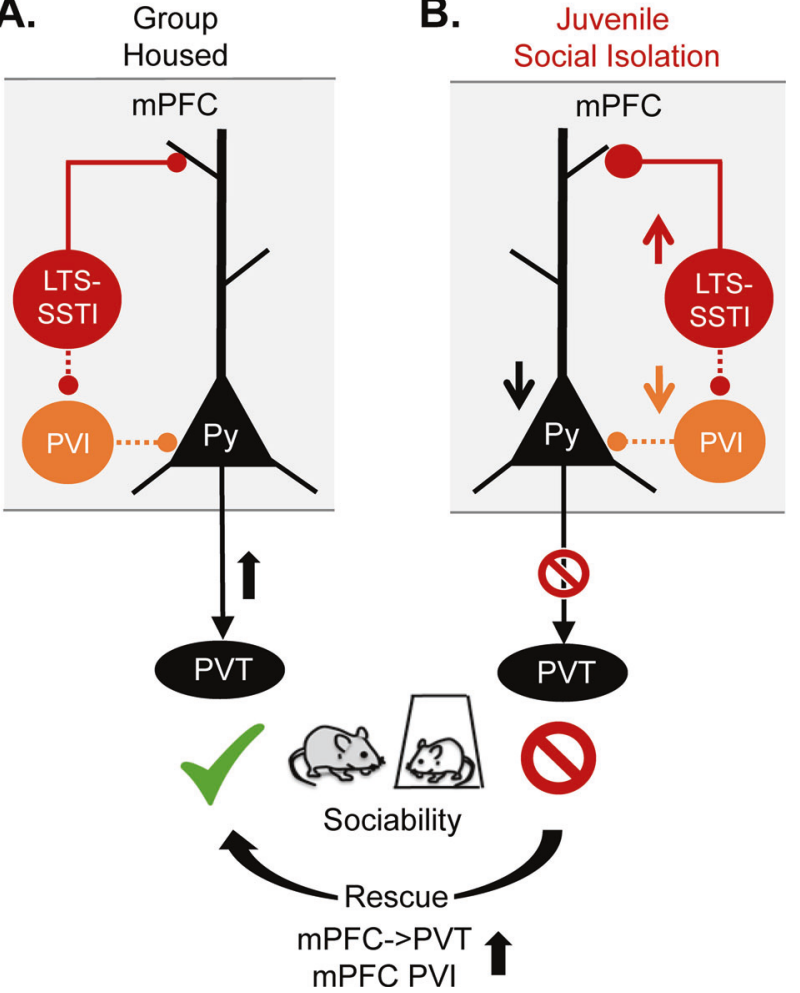

Fig. 1 A vulnerable mPFC social circuit in response to juvenile social isolation in mice. A. Activation of $\mathrm{mPFC} \rightarrow \mathrm{PVT}$ projection neurons (black) or mPFC PVIs (orange) is essential for normal sociability in adult group-housed mice. B. However, these neurons show decreased intrinsic excitability and an increased inhibitory input drive from mPFC LTS-SSTIs (red) in juvenile socially isolated (jSI) mice, which show decreased sociability in adulthood. Decreased sociability can be induced in normal animals by inhibiting $\mathrm{mPFC} \rightarrow \mathrm{PVT}$ projection/mPFC PVIs or activating mPFC LTS-SSTIs in adulthood. Sociability deficits of jSI mice can be rescued by increasing PFC $\rightarrow$ PVT projection neuron or mPFC-PVI activity (bottom arrow).

\footnotetext{
${ }^{1}$ Department of Psychiatry, Icahn School of Medicine at Mount Sinai, New York, NY, USA; ${ }^{2}$ Nash Family Department of Neuroscience, Icahn School of Medicine at Mount Sinai, New York, NY, USA; ${ }^{3}$ Department of Ophthalmology, Icahn School of Medicine at Mount Sinai, New York, NY, USA; ${ }^{4}$ Mindich Child Health and Development Institute, Icahn School of Medicine at Mount Sinai, New York, NY, USA and ${ }^{5}$ Friedman Brain Institute, Icahn School of Medicine at Mount Sinai, New York, NY, USA

Correspondence: Hirofumi Morishita (hirofumi.morishita@mssm.edu)

${ }^{6}$ Present address: One Gustave L. Levy Place, Box 1230, New York, NY 10029, USA
}

Published online: 1 September 2020 
observed social deficits may be caused not by lack of social experience per se, but by adaptations to living in isolation that prevent mice from adapting to group living following re-housing. The juvenile window may be a sensitive period for behavioral plasticity, and once closed, mice may not be able to adjust their social strategy.

Our studies also demonstrated that the identified vulnerable mPFC social circuit is a promising target for treatments of social behavior deficits. Through stimulation of $\mathrm{mPFC} \rightarrow$ PVT neurons [5] or mPFC PVIs in adulthood [3], we are able to rescue the sociability deficits caused by jSI. Given that previous genetic and transcriptomic studies show that many risk genes for autism and schizophrenia are highly expressed in the identified vulnerable social circuit, future studies are warranted to investigate the extent to which disease risk genes impact maturation of the identified circuit, and the effect of targeting the circuit for treating social processing deficits relevant to psychiatric disorders.

\section{FUNDING AND DISCLOSURE}

This work was supported by NIH R01MH118297, R01MH119523, and the Simons Foundation/SFARI (610850). The author acknowledges the co-authors on the original papers L.K. Bicks, S. Akabarian, K. Yamamuro, and M. Leventhal. The author declares no competing interests.

\section{ADDITIONAL INFORMATION}

Publisher's note Springer Nature remains neutral with regard to jurisdictional claims in published maps and institutional affiliations.

\section{REFERENCES}

1. Pitman A, Mann F, Johnson S. Advancing our understanding of loneliness and mental health problems in young people. Lancet Psychiatry. 2018;5:955-6. https:// doi.org/10.1016/S2215-0366(18)30436-X.

2. Makinodan M, Rosen KM, Ito $S$, Corfas G. A critical period for social experiencedependent oligodendrocyte maturation and myelination. Science. 2012;337:1357-60. https://doi.org/10.1126/science.1220845.

3. Bicks LK, Yamamuro K, Flanigan ME, Kim JM, Kato D, Lucas EK et al. Prefrontal parvalbumin interneurons require juvenile social experience to establish adult social behavior. Nat Commun. 2020;11:1003. https://doi.org/10.1038/s41467-020-14740-z.

4. Bicks LK, Koike H, Akbarian S, Morishita H. Prefrontal cortex and social cognition in mouse and man. Front Psychol. 2015;6:1805. https://doi.org/10.3389/fpsyg.2015.01805.

5. Yamamuro K, Bicks LK, Leventhal MB, Kato D, Im S, Flanigan ME et al. A prefrontal-paraventricular thalamus circuit requires juvenile social experience to regulate adult sociability in mice. Nat Neurosci. 2020. https://doi.org/10.1038/ s41593-020-0695-6.

6. Yamamuro K, Yoshino H, Ogawa Y, Makinodan M, Toritsuka M, Yamashita M et al. Social isolation during the critical period reduces synaptic and intrinsic excitability of a subtype of pyramidal cell in mouse prefrontal cortex. Cereb Cortex. 2018;28:998-1010. https://doi.org/10.1093/cercor/bhx010. 